\title{
Diagnosis of Ovarian
}

Metastasis from Gestational

Trophoblastic Neoplasia by 3D

Power Doppler Ultrasound and

Dynamic Contrast-Enhanced

Magnetic Resonance Imaging:

Case Report

\author{
E. Araujo Júnior S.Y. Sun F.F. Campanharo \\ D.C. Nacaratto L.M.M. Nardozza R. Mattar V.V. Habib
}

A.F. Moron

Department of Obstetrics, Federal University of São Paulo (UNIFESP),

São Paulo, Brazil

\section{Key Words}

Ovarian - Metastasis - Gestational trophoblastic neoplasia - Three-dimensional ultrasound $\cdot$ Magnetic resonance imaging

\begin{abstract}
Choriocarcinoma is a highly malignant disease of trophoblastic cells, which affects young women in the reproductive years. The main sites of metastasis from choriocarcinoma are lung, vagina, liver, gastrointestinal tract and kidneys, and the involvement of the ovaries is extremely rare. The diagnosis of ovarian metastasis is made mainly by two-dimensional ultrasonography (2DUS) with color Doppler, which shows a large vessel in the center of the mass. The three-dimensional power Doppler ultrasound (3D power Doppler) and dynamic contrast-enhanced magnetic resonance imaging (MRI) are new diagnostic modalities not yet published in literature. We report a case of metastatic choriocarcinoma with left ovary involvement in a 48-year-old woman with history of molar pregnancy and irregular follow-up of this disease. We emphasize the main findings by 2DUS with color Doppler, 3D power Doppler and dynamic contrast-enhanced MRI. 3D power Doppler is able to improve the assessment of anatomical relationships of vessels with the ovarian mass, while the resonance angiography allows us to evaluate the anatomic relations of the mass and adjacent structures, as well as the iliac vessels. The 3D power Doppler and dynamic contrast-
\end{abstract}

Prof. Luciano M.M. Nardozza, PhD Department of Obstetrics, Federal University of São Paulo (UNIFESP) 
enhanced MRI are promising methods in the evaluation of metastasis arising from gestational trophoblastic tumors.

\section{Introduction}

The gestational trophoblastic disease consists of two types of molar pregnancies: complete hydatidiform mole and partial hydatidiform mole. Both have the potential for transformation into gestational trophoblastic neoplasia (GTN). Although most cases of GTN came from molar pregnancy, there are a few cases of choriocarcinoma arising from non-molar pregnancies [1]. The incidence of hydatidiform mole in Asia is 70 to 10 times higher than in Europe or North America, occurring mostly at the extremes of reproductive life [1]. Women over age 40 are 50 to 10 times more likely to have molar pregnancy than younger women [2].

Two-dimensional ultrasound (2DUS) is the most frequently used imaging method for the diagnosis of hydatidiform mole. The most common finding in the first trimester of complete mole is the presence of a predominantly echogenic mass with heterogeneous aspect inside the uterine cavity and hypoechoic areas around it, through transvaginal approach. This initial appearance is nonspecific and can be confused with incomplete miscarriage [3]. On the other hand, partial mole presents with a large placenta compared to the uterine cavity, with hypoechoic areas diffusely distributed. There is presence of an embryo or fetus with congenital anomalies, which may be alive, but generally with growth restriction or dead.

Approximately $18-28 \%$ of complete hydatidiform moles and $2-4 \%$ of partial hydatidiform moles become GTN [1]. GTN metastases can occur anywhere in the body and the organs most commonly involved are the lung, vagina, liver, gastrointestinal tract and kidneys [4]. Atypical locations of metastatic chorioangioma have been described for the skin and eyes $[5,6]$. Metastases in the ovaries are not uncommon, and $50-20 \%$ of neoplasias in these organs are from other sites [7]; however, there are no descriptions in the literature of ovarian metastases from GTN. The differentiation between primary tumor and metastasis is of great importance because the clinical management of both is different [8].

The diagnosis of metastasis is usually performed by 2DUS in association with color Doppler [9]. There are descriptions in the literature of using three-dimensional power Doppler (3D power Doppler) and dynamic contrast-enhanced magnetic resonance imaging (MRI) in the diagnosis of ovarian metastasis.

The aim is to describe a case of ovarian metastasis from a choriocarcinoma, showing the applications of new imaging technologies such as 3D power Doppler and dynamic contrast-enhanced MRI.

\section{Case Report}

A 48-year-old woman with 12 pregnancies and 10 deliveries was admitted to our service in July 2005 with a complaint of 11 weeks of missed menses, intermittent vaginal bleeding for a month and a blood test for $\beta$-HCG positive. Physical examination showed the uterus soft and extended to 18 weeks. 
The 2DUS vaginally demonstrated increased size of the uterus $(1,700 \mathrm{ml})$, with heterogeneous and hyperechoic contents inside, with hypoechoic oval images, suggestive of gestational trophoblastic disease. The quantitative $\beta$-HCG was $>500,000 \mathrm{mIU} / \mathrm{ml}$ and free T4 was $2.9 \mathrm{ng} / \mathrm{dl}$. She underwent uterine vacuum aspiration. Histopathologic examination revealed complete hydatidiform mole. She evolved with progressive decrease of $\beta$-HCG levels, which, however, remained positive 2 months after the initial procedure, which brought us to indicate a hysterectomy. After this procedure, histopathological examination revealed focal superficial endomyometrial permeation by groups of trophoblastic cells with standard, atypical, without, however, characteristic formation of chorionic villi (invasive mole). After two and a half months of surgery, the patient was asymptomatic, with levels of $\beta$-HCG negative $(<5 \mathrm{mUI} / \mathrm{ml})$ and remained in outpatient follow-up.

From July to August 2007, a slow and progressive increase in the levels of $\beta$-HCG began and the patient was submitted to the staging of the disease by MRI of the abdomen, pelvis, and skull, in addition to computed tomography (CT) of the chest, and all these tests were negative. She began mono-chemotherapy with methotrexate, a total of 5 cycles between August and October 2008, with a satisfactory response. At this time, it was decided to change the chemotherapy regimen with the addition of actinomycin $\mathrm{D}$, when the woman was released from the service.

The patient returned to service in October 2011 presenting with cough, dyspnea, daily fever up to $38.5^{\circ} \mathrm{C}$ and pain in the left iliac fossa and was hospitalized for investigation, support and restaging. Lab tests showed anemia $(\mathrm{Hb}=8.1 / \mathrm{Ht}=25)$, leukocytosis of 10,200 with 7,681 neutrophils, $\mathrm{C}$ reactive protein measuring 123 , blood and urine cultures were negative and $\beta$-HCG was $>200,000$ $\mathrm{mIU} / \mathrm{ml}$. We opted for broad-spectrum antibiotic coverage aimed at the pulmonary infection and transfusion of two units of red blood cells. The CT imaging showed the presence of a $10 \times 7 \mathrm{~cm}$ mass in the left lower lobe of the lung with atelectasis and underlying pleural effusion and multiple lesions in the liver and kidneys that were suggestive of metastasis (fig. 1). In the pelvis, the vaginal 2DUS showed the presence of a hypoechoic solid mass in close contact with the vaginal vault, measuring 7.1 $\times 6.4 \times 7.2 \mathrm{~cm}$ ( fig. 2a). The 2DUS mode power Doppler showed a large vessel that entered the center of the mass and had low levels of resistance index $(\mathrm{RI}=0.25)$ and pulsatility index $(\mathrm{PI}=0.30)$. The 3D power Doppler was performed with an endocavitary volumetric probe (RIC 6-9) of the device Voluson 730 Expert (General Electric Medical Systems, Zipf, Austria) which allowed an assessment of spatial relationships of the mass with their vessels, confirming the rich vascularization of the mass ( $\underline{\text { fig. } 3 \mathrm{a}}$ and b). MRI was performed on the device Sonata Maestro Class $1.5 \mathrm{~T}$ (Siemens, Erlangen, Germany) with dedicated 8-channel coils. MRI was performed with the technique turbo spin-echo (TSE), gradient-echo (FLASH) $\mathrm{T}_{1}$-weighted and $\mathrm{T}_{2}$ images were obtained in axial and coronal planes. Sequences were performed 'in-phase' and 'out-of-phase', and fat saturation techniques and acquisitions after injection of paramagnetic contrast was performed. MRI revealed avoluminous solid expansive formation with heterogeneous change with contrast in the topography of the uterus and left adnexal region, in close contact with the dome of the anterior vagina and measuring $8.8 \times 7.7 \mathrm{~cm}$ (fig, 4a). After the injection of contrast, the dynamic contrast-enhanced MRI clearly showed the rich vascularity of the mass, in addition to its relationship with the great vessels of the pelvis (fig. $4 \mathrm{~b}$ ). The right ovary had dimensions of $2.7 \times 2.5 \mathrm{~cm}$, shape, contours and normal intensity of signals.

The woman was restaged with stage IV disease, high risk, with a failed methotrexate and actinomycin D chemotherapy, and fever caused by the tumor. Those signs indicated multidrug therapy regimen (etoposide, methotrexate, actinomycin D, cyclophosphamide and vincristine - EMACO) with clinical improvement after 5 cycles performed and progressive decrease of $\beta$-HCG levels. She was in follow-up at the Pregnancy Neoplasia Sector (Department of Obstetrics) and Clinical Oncology (Department of Medical Clinic) of the Federal University of São Paulo, but she was lost to follow-up again. The loss to follow-up makes it impossible to assess the 2D/3D ultrasound images and $\beta$-HCG level of the patient.

\section{Discussion}

Choriocarcinoma is a highly malignant neoplasia of trophoblastic cells, which affects young women of childbearing age. Most choriocarcinoma (50\%) are following molar pregnancies; however, miscarriage and ectopic pregnancy are responsible for $25 \%$ each [10]. The symptoms of choriocarcinoma are confused with those of a hydatidiform 
mole like persistent genital bleeding, uterus larger than expected for gestational age, hyperemesis and early preeclampsia. The predictors for choriocarcinoma, after hydatidiform complete mole, are: blood levels of $\beta$-HCG greater than $100,000 \mathrm{mIU} / \mathrm{ml}$, theca lutein cysts larger than $6.0 \mathrm{~cm}$, enlarged uterus for gestational age and maternal age greater than 40 years [11]. The choriocarcinoma grows rapidly, has early metastasis and the main site is the lungs, but liver and vagina are also frequently affected [12]. In our case, the diagnosis of choriocarcinoma was realized in the histopathological findings of invasive mole, increase of $\beta$-HCG level after the hysterectomy, absence of response in mono-chemotherapy, and quick evolution to metastasis of lungs, kidneys and liver.

We concluded that the mass originated from the ovary because the patient had previous hysterectomy and the MRI evidenced a normal right ovary. The mass was located in the left adnexal region in intimate contact with the external surface of the uterus. Furthermore, the mass presented a large vessel ('lead vessel') that entered in its center and it showed low levels of resistance and pulsatility indices. In a study by Testa et al. [13] observed that the 'lead vessel' was present in 11 of $31(35.2 \%)$ ovarian metastases, but in only 2 of 102 primary ovarian tumors $(0.01 \%)$.

In our case, the patient stopped the follow-up of the disease and only returned to service after three years, presenting as disseminated disease with metastasis in lungs, liver and kidneys. We also observed the presence of a solid left ovarian mass with 2DUS whose examination with color Doppler showed the presence of a large vessel in the center of the mass with low resistance and pulsatility indices. We also used the new diagnostic modalities 3D power Doppler and dynamic contrast-enhanced MRI techniques not yet published in the literature for the evaluation of ovarian metastasis. The 3D power Doppler allowed an assessment of the anatomic relation between vessels and the ovarian mass, proving to be a highly vascularized mass. The potential advantage of power Doppler is the ability to detect flow in small vessels as the vessels of the placental intervillous space [14]. This advantage is particularly important in the evaluation of ovarian metastasis, because the neo-vessels have a small caliber. MRI is considered a routine examination to assess the local extent of tumor and to search for distant metastasis in cases of GTN [15]; however, its use for the evaluation of ovarian metastasis has not been described in the literature yet. Dynamic contrast-enhanced MRI allowed us to assess the relationship of the mass and adjacent structures, as well as the iliac vessels, proving they are not compromised.

In summary, the 3D power Doppler and dynamic contrast-enhanced MRI are important adjunctive methods to evaluate the characteristics of a mass, as well as its anatomic relations to adjacent structures. These two imaging methods appear to offer a great improvement in the assessment of metastasis arising from gestational trophoblastic tumors. 

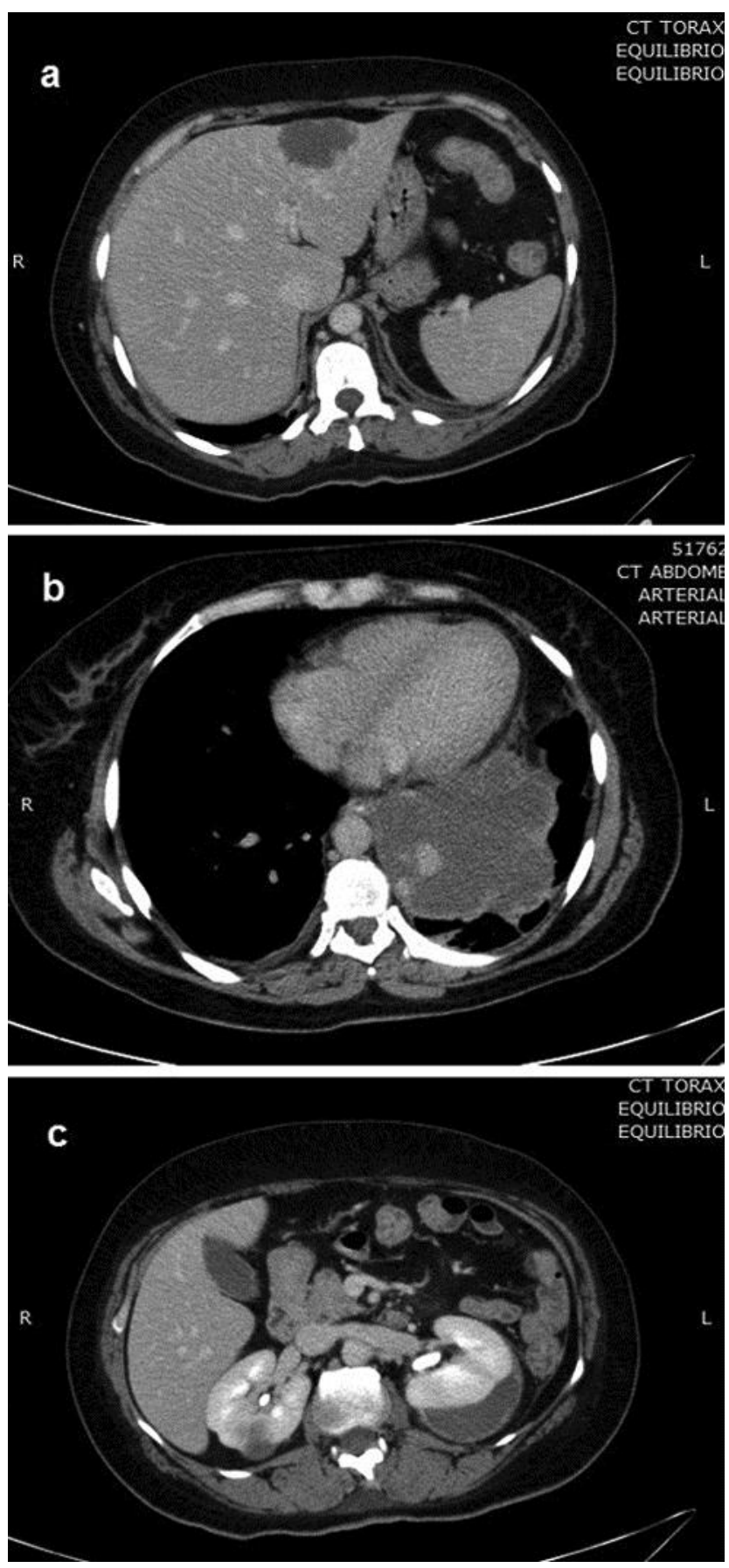

Fig. 1. CT in axial plane after intravenous contrast of abdomen showing the metastasis (hyperechoic lesions) to liver (a), lung (b) and kidneys (c). 

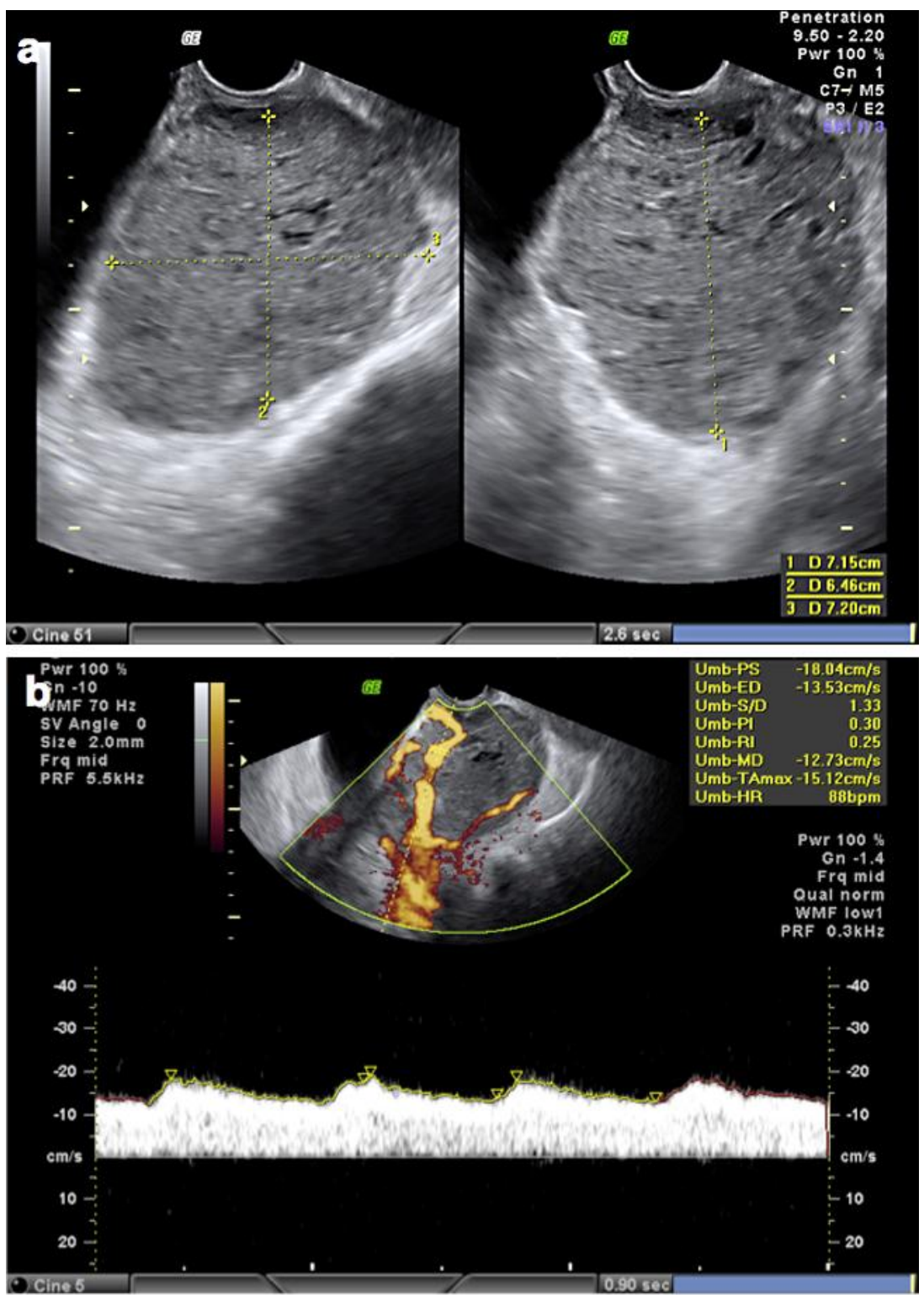

Fig. 2. a Measurement of ovarian tumor by 2DUS. b 2DUS with color Doppler showing the 'lead vessel', and spectral Doppler proving the low indices of resistance (pulsatiliy and resistance indices) of ovarian tumor vascularization. 

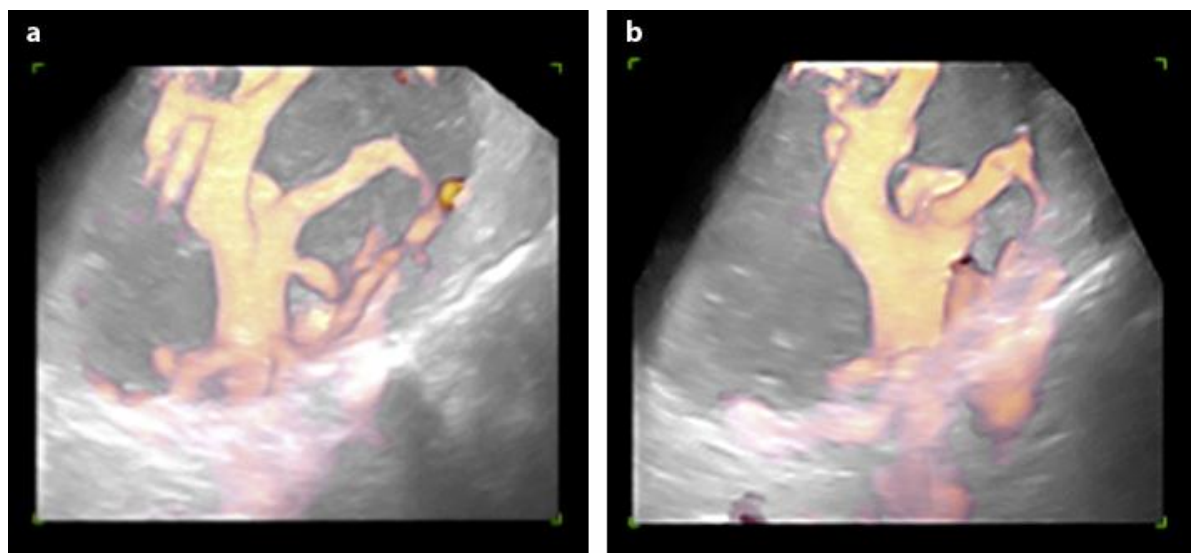

Fig. 3. a, b 3D power Doppler showing the high vascularization of ovarian tumor.
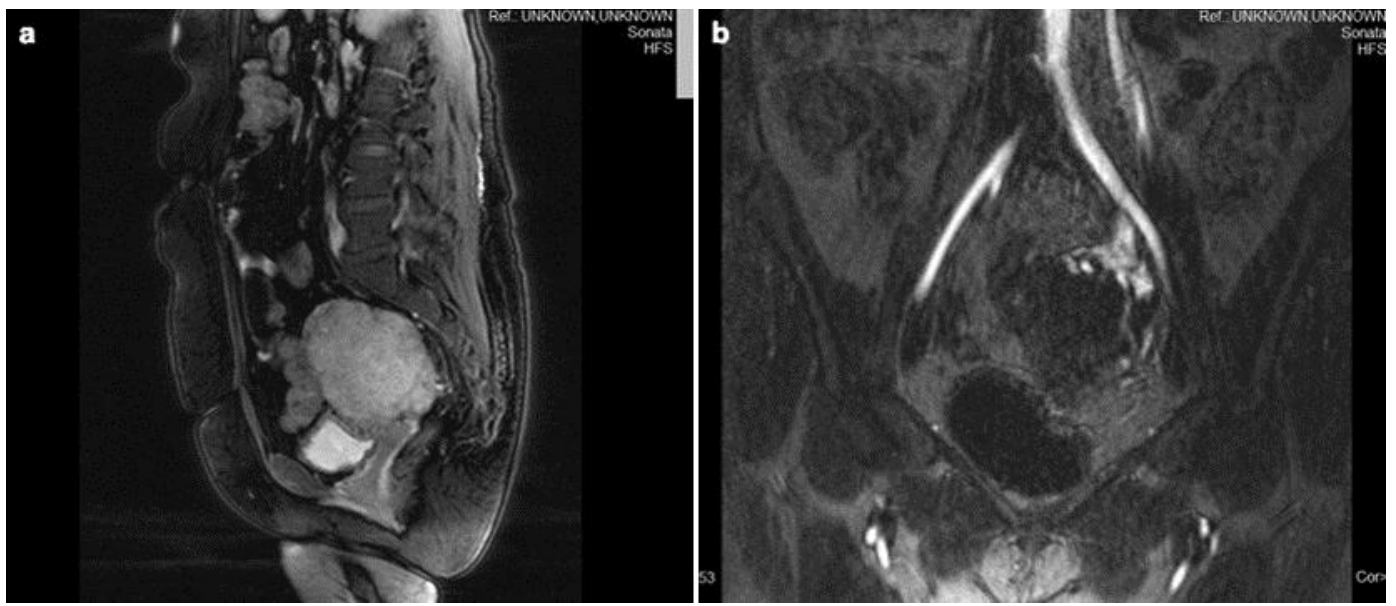

Fig. 4. a MRI in $\mathrm{T}_{2}$-weighted sequence in sagittal plane showing the relationship of ovarian tumor with adjacent structures. b MRI with paramagnetic contrast (dynamic contrast-enhanced MRI) showing the vascularization of ovarian tumor and its relationships with iliac vessels.

\section{References}

1 Garner EIO, Goldstein DP, Feltmate CM, Berkowitz RS: Gestational trophoblastic disease. Clin Obstet Gynecol 2007;50:112-122.

-2 Palmer JR: Advances in the epidemiology of gestational trophoblastic disease. J Reprood Med 1994;39:155-162.

-3 Fowler DJ, Lindsay I, Seckl MJ, Sebire NJ: Routine pre-evacuation ultrasound diagnosis of hydatidiform mole: experience of more than 1,000 cases from a regional referral center. Ultrasound Obstet Gynecol 2006;27:56-60.

4 Bagshawe KD: Trophoblastic tumors; in Coppleson M (ed): Gynecologic Oncology. 2nd edition. Edinburgh, Churchill Livingstone, 1992, pp 1013-1046.

-5 Gangadharan VP, Prakash NP, Chitrathara K, Sasidharan K, Ramachandran K: Ocular metastasis of choriocarcinoma. Br J Radiol 1999;72:1216-1217.

6 Chama CM, Nggada HA, Nuhu A: Cutaneous metastasis of gestational choriocarcinoma. Int J Obstet Gynecol 2002;77:249-250. 
7 Young RH, Scully RE: Metastatic tumors in the ovary: a problem-oriented approach and review of the recent literature. Semin Diagn Pathol 1991;8:250-276.

8 Yada-Hashimoto N, Yamamoto T, Kamiura S, Seino H, Ohira H, Sawai K, Kimura T, Saji F: Metastatic ovarian tumors: a review of 64 cases. Gynecol Oncol 2003;89:314-317.

-9 Alcázar JL, Galán MJ, Ceamanos C, García-Manero M: Transvaginal gray scale and color Doppler sonography in primary ovarian cancer and metastatic tumors to the ovary. J Ultrasound Med 2003;22:243-247.

10 Wagner BJ, Woodward PJ, Dickey GE: Gestational trophoblastic disease: radiologic-pathologic correlation. Radiographics 1996;16:131-148.

11 Maestá I, Rudge MV, Abreu ES, Dalben I, Peraçoli JC: [Clinical and histopathological predictors of gestational trophoblastic tumor after complete hydatidiform mole] [article in Portuguese]. Rev Bras Ginecol Obstet 2000;22:167-173.

12 Vijay RK, Kaduthodil MJ, Bottomley JR, Abdi S: Metastatic gestational trophoblastic tumour presenting as spontaneous subcapsular renal haematoma. Br J Radiol 2008;81:e234-e237.

13 Testa AC, Mancari R, Di Legge A, Mascilini F, Salutari V, Scambia G, Ferrandina G: The 'lead vessel': a vascular ultrasound feature of metastasis in the ovaries. Ultrasound Obstet Gynecol 2008;31:218-221.

$14 \mathrm{Yu} \mathrm{CH}$, Chang CH, Ko HC, Chen WC, Chang FM: Assessment of placental fractional moving blood volume using quantitative three-dimensional power Doppler ultrasound. Ultrasound Med Biol 2003;29:19-23.

15 Ohmaru T, Yamakawa H, Netsu S, Nokubi M, Konno R: Placental site trophoblastic tumor (PSTT) with multiple metastases and extremely poor prognosis. Int J Clin Oncol 2009;14:452-456. 